\title{
High-Density Modification of H-Terminated Si(111) Surfaces Using Short-Chain Alkynes
}

\author{
Sidharam P. Pujari, ${ }^{\dagger}$ Alexei D. Filippov, ${ }^{\dagger}$ Satesh Gangarapu, ${ }^{\dagger}$ and Han Zuilhof* ${ }^{\dagger}{ }^{\dagger}, \S_{(0)}$ \\ ${ }^{\dagger}$ Laboratory of Organic Chemistry, Wageningen University, Stippeneng 4, 6708 WE Wageningen, The Netherlands \\ ${ }^{\ddagger}$ School of Pharmaceutical Sciences and Technology, Tianjin University, 92 Weijin Road, Tianjin, People’s Republic of China \\ ${ }^{\S}$ Department of Chemical and Materials Engineering, King Abdulaziz University, Jeddah, Saudi Arabia
}

\section{Supporting Information}

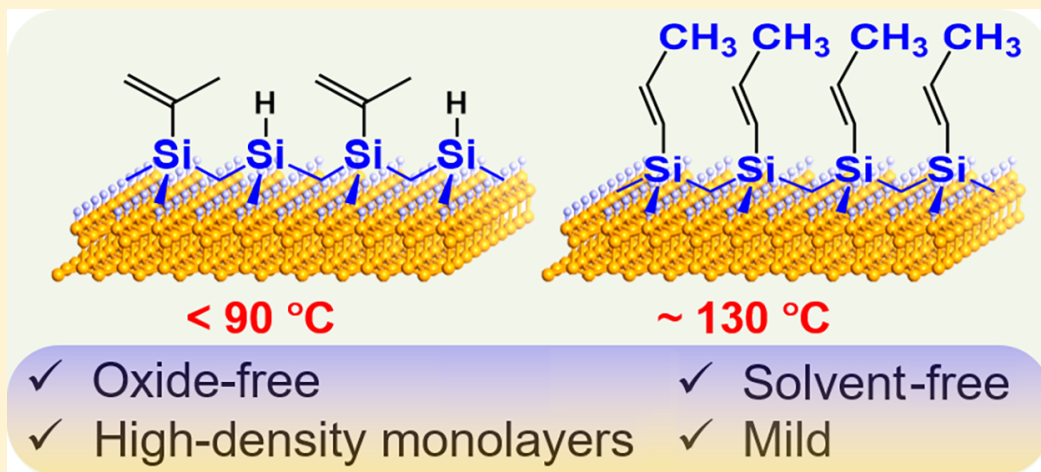

ABSTRACT: $\mathrm{H}-\mathrm{Si}(111)$-terminated surfaces were alkenylated via two routes: through a novel one-step gas-phase hydrosilylation reaction with short alkynes $\left(\mathrm{C}_{3}\right.$ to $\left.\mathrm{C}_{6}\right)$ and for comparison via a two-step chlorination and Grignard alkenylation process. All modified surfaces were characterized by static water contact angles and X-ray photoelectron spectroscopy (XPS). Propenyl- and butenyl-coated $\mathrm{Si}(111)$ surfaces display a significantly higher packing density than conventional $\mathrm{C}_{10}-\mathrm{C}_{18}$ alkynederived monolayers, showing the potential of this approach. In addition, propyne chemisorption proceeds via either of two approaches: the standard hydrosilylation at the terminal carbon (lin) at temperatures above $90{ }^{\circ} \mathrm{C}$ and an unprecedented reaction at the second carbon (iso) at temperatures below $90{ }^{\circ} \mathrm{C}$. Molecular modeling revealed that the packing energy of a monolayer bonded at the second carbon is significantly more favorable, which drives iso-attachment, with a dense packing of surface-bound iso-propenyl chains at $40 \%$ surface coverage, in line with the experiments at $<90{ }^{\circ} \mathrm{C}$. The highest density monolayers are obtained at $130{ }^{\circ} \mathrm{C}$ and show a linear attachment of 1-propenyl chains with $92 \%$ surface coverage.

\section{INTRODUCTION}

Since the seminal work by Linford and Chidsey, ${ }^{1}$ covalently bound organic monolayers on the $\mathrm{Si}(111)$ crystal face have proven to be a topic of continued intense interest in both fundamental and applied chemistry. ${ }^{2-6}$ Organic molecules bound to silicon through the $\mathrm{Si}-\mathrm{C}$ bond are of special significance because they allow the fabrication of Si surfaces lacking the interfacial $\mathrm{SiOx}$ surface species that lead to electronic defects and reduced stability. Oxide-free covalent modification of the $\mathrm{Si}$ surface therefore holds great promise in improving the performance of silicon-based semiconductors through electrical and chemical passivation. ${ }^{7,8}$ Furthermore, oxide-free modified Si surfaces offer the added possibility to fine-tune semiconductor behavior by coupling organic molecules to the surface, giving rise to e.g. bottom-up molecular electronics. $^{2,9,10}$ Moreover, stable, covalently modified oxidefree $\mathrm{Si}$ surfaces have many applications in the immobilization and detection of biomolecules, ${ }^{11-13}$ offer a platform on which fundamental physical and chemical processes such as electron transfer and reactivity can be studied, ${ }^{14}$ and are useful in molecular catalysis. ${ }^{15}$

Over the past two decades, covalently bound monolayers on $\mathrm{Si}(111)$ have been produced by research groups around the world through a range of methods, including the hydrosilylation of alkenes and alkynes induced upon thermal activation, $, 1,16$ photochemical activation, ${ }^{12,17-21}$ or radical activation. ${ }^{22}$ Alternative methods include two-step chlorination/alkylation procedures involving Grignard reagents ${ }^{23-26}$ and electrochemical grafting. ${ }^{27}$ These procedures have yielded dense, high-quality monolayers that are highly stable in air, in water (for even over a year), and a range of $\mathrm{pH}$ values and temperatures. ${ }^{24,28-30}$ The most frequently used of these methods is the hydrosilylation of H-terminated Si surfaces by terminally unsaturated alkenes or alkynes, typically $\mathrm{C}_{8}$ or longer, as the reaction conditions are typically mild and the

Received: October 23, 2017

Revised: November 29, 2017

Published: December 14, 2017 
reproducibility of the formed monolayers is very high. However, the most densely packed monolayers have been obtained by the chlorination-Grignard route (with up to $100 \%$ packing density for $\mathrm{CH}_{3}$-terminated monolayers), which is highly advantageous for electronic studies. ${ }^{3}$ With this being the state of the art, there are thus still two significant limitations in this field: (1) Mild methods do not yet exist for short-chain monolayers: the superbly passivated surfaces with short chains have only been prepared, pioneered by the Lewis group, using Grignard chemistry. ${ }^{3}$ However, harsh reaction conditions are required to achieve this including the initial chlorination of $\mathrm{Si}$ surface using $\mathrm{PCl}_{5} \cdot{ }^{23,25}$ Moreover, wet chemistry involving highly reactive chemicals such as Grignard reagents is difficult to perform at a large scale. ${ }^{31}$ These conditions also strongly limit the possible terminal groups that can be attached. If additional functionalization of the monolayer is desired, then subsequent reaction steps need to be performed, inevitably yielding a more heterogeneous surface (functionalization rarely proceed with a $100 \%$ yield $)^{32}$ and surface defects. (2) All methods that currently produce high-quality $\mathrm{Si}-\mathrm{C}$ bonded monolayers, even the very mild ones, use wet chemistry. This invokes some limitations, specifically regarding the atom efficiency of the reactions (production of waste). In addition, solution-based methods are only useful for preparation of monolayers with relatively long chain lengths, i.e., with sufficiently high boiling points: monolayers derived from 1alkenes/1-alkynes with short chains (typically $<\mathrm{C}_{8}$ ) cannot be routinely prepared via these methods. ${ }^{3,23,25}$ Finally, the batchwise wet-chemical preparation hampers scalability, i.e., making hundreds of high-quality functionalized wafers.

To take advantage of the mild hydrosilylation while overcoming the limitations of wet chemistry concerning chain length and solvent use, we therefore report here a new approach to modify $\mathrm{H}-\mathrm{Si}(111)$ with gaseous alkynes at elevated pressures. Propyne, 1-butyne, 1-pentyne, and 1-hexyne were used for surface modification. The produced monolayers were characterized by ellipsometry, static contact angle (SCA), and X-ray photoelectron spectroscopy (XPS) measurements. XPS C 1s spectra were simulated quantum chemically using a B3LYP/6-311G(d,p)-based method that has previously been shown to be reliable. ${ }^{33-35}$

In all hydrosilylation reactions of alkynes onto hydrogenterminated Si surfaces up to now, the reactivity exclusively takes place at the terminal carbon atom. Since our current studies revealed an unprecedented iso rather than linear reactivity for especially the shortest chains, we studied the organization and interactions of short chains in the resulting monolayers using molecular mechanics approaches in a periodic box setting, together with an estimation of the binding energy of alkynes with the $\mathrm{H}-\mathrm{Si}(111)$ surface using quantum chemical G3 calculations. The combination of these efforts outlines a novel mild approach to $\mathrm{Si}$ surface modifications and reveals the driving forces for linear versus iso attachment.

\section{EXPERIMENTAL SECTION}

Materials. Ammonium fluoride $\left(\mathrm{NH}_{4} \mathrm{~F}, 40 \%\right.$ in water, semiconductor grade VLSI PURANALTM Honeywell 17767), acetone (semiconductor grade VLSI PURANALTM Honeywell 17617), chlorobenzene (CHROMASOLV 99.9\% for HPLC), tetrahydrofuran (THF, CHROMASOLV $\geq 99.9 \%$ for HPLC, inhibitor free), pentane (HPLC grade, 99\%) dichloromethane (DCM, CHROMASOLV $\geq 99.8 \%$ for HPLC), propyne (C3YNE, $\geq 99 \%$, in Sure-Pac), 1-butyne (C4YNE, $\geq 98 \%$, in Sure-Pac), 1-pentyne (C5YNE, 99\%), 1-hexyne (C6YNE, 97\%), 1-propenylmagnesium bromide in THF (0.5 M), iso- propenylmagnesium bromide in THF $(0.5 \mathrm{M})$, phosphorus pentachloride $\left(\mathrm{PCl}_{5}, \geq 98 \%\right)$, and benzoyl peroxide $(\geq 98 \%)$ were obtained from Sigma-Aldrich. 1-Butyne (C4YNE, $\geq 98 \%$, in cylinder) also obtained from ABCR Germany. Argon (Argon, 6.0 HiQ) was purchased from Linde Gas. 1-Pentyne was degassed by three subsequent freeze-pump-thaw cycles and stored in an argon-purged glovebox. 1-Hexyne and chlorobenzene were purified by distillation at reduced pressure, degassed by three freeze-pump-thaw cycles, and then stored in an argon-purged glovebox. Dichloromethane and tetrahydrofuran were purified by distillation in a PureSolv 400 setup from Innovative Technology Inc., USA; these solvents were dispensed under argon flow into oven-dried, vacuum evacuated, and subsequently argon-purged glassware. All other chemicals were used as received. TX761 Alpha Swabs were purchased from Texwipe, USA. Single-side polished, n-type phosphorus-doped silicon (111) wafers were obtained from Siltronix, France. The wafers have a miscut angle of $0.2^{\circ}$ toward $\langle 11-2\rangle$ orientation, thickness $500-550 \mu \mathrm{m}$, and a resistivity of $1-10 \Omega \mathrm{cm}$. Deionized water with a conductivity of 18.2 $\mathrm{M} \Omega \mathrm{cm}$ was taken from a Milli-Q Integral 3 System water purification setup with a Q-POD dispenser.

Methods. Preparation of H-Terminated Si(111). H-Si(111) surfaces were prepared in a wet-chemical etching procedure as previously reported. ${ }^{36}$ Silicon wafers were cut into $1 \times 1 \mathrm{~cm}$ pieces and sonicated in acetone, methanol, and dichloromethane for each $5 \mathrm{~min}$. The substrates were rinsed with flowing acetone and subsequently dried in a jet of argon; this was repeated until the surfaces appeared as homogeneously reflective dust-free mirrors. The surfaces were then oxidized for $10 \mathrm{~min}$ in air plasma using a PDC-002 (plasma cleaner) and a PDC-FMG-2 (plasmaFlo) gas flow mixer (both: Harrick Scientific Products, Inc., Ossining, NY). The plasma chamber was pumped down to less than $(1.2 \pm 0.2) \times 10^{-2}$ mbar prior to the introduction of air plasma. Pieces of silicon substrates were placed in the plasma cleaner, and surface oxidation was achieved using an 0.3 SCFH (standard cubic feet per hour) air flow and a power of $29.6 \mathrm{~W}$ at $300 \mathrm{~m}$ Torr pressure. The samples were taken out, then quickly but thoroughly rinsed with copious amounts of deionized water, and dried in an argon jet. Subsequently, the substrates were etched in an argonsaturated $40 \%$ aqueous ammonium fluoride solution for $15 \mathrm{~min}$. The surfaces were taken out and again rinsed with water and dried with a stream of argon. The $\mathrm{H}-\mathrm{Si}(111)$ surfaces were immediately (i.e., within 30-60 s after taking them out of the $\mathrm{NH}_{4} \mathrm{~F}$ solution) used for either further modification or characterization.

Gas-Phase Modification of H-Si(111) Surfaces. The reaction of C3YNE or C4YNE with $\mathrm{H}-\mathrm{Si}(111)$ was done in a $10 \mathrm{~mL}$ cylindrical stainless-steel high pressure reactor (Parr Instrument Company; Series 2500 Micro Batch System; see Supporting Information Figure S2), which is equipped with a temperature controller for heating the contents of the reactor. The reaction vessel was cleaned in air plasma (Harrick Scientific Products, Inc., Pleasantville, NY) for $1 \mathrm{~h}$ prior to the reaction; this turned out to be critical for good monolayer formation. A vacuum of 5 mbar was then applied to both the reactor and the steel connections, which were kept at $200{ }^{\circ} \mathrm{C}$ overnight to remove any contamination. Freshly prepared $\mathrm{H}-\mathrm{Si}(111)$ samples were placed in a reaction vessel into which a thermocouple was fitted as shown in Scheme 1. The vessel was then immediately connected to a metallic pipeline with a tightly fitting adapter through which a strong stream of argon was flowing. The vessel and adapter were clamped

Scheme 1. Schematic of Experimental Reactor Setup

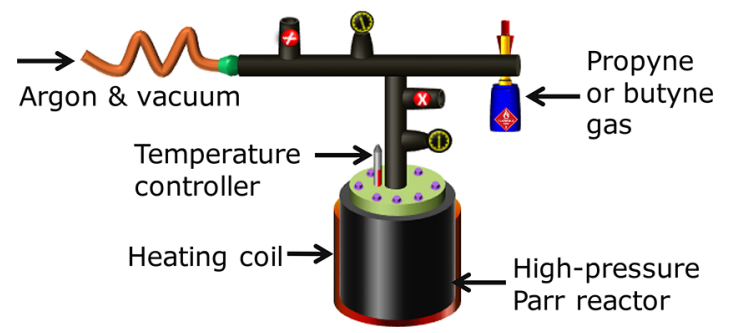


tightly so that no gas exchange between vessel and surroundings was possible. Argon was removed by means of a vacuum pump (Edwards XDS 10), and the resulting pressure in the system was 5 mbar. The cooled Parr reactor and C3YNE gas tank are connected with two valves. The process consists of two steps: (1) cooling of the Parr reactor using the liquid nitrogen bath under high vacuum; (2) condensation of C3YNE gas in the cooled Parr reactor. The first valve was closed when the temperature reaches $-10{ }^{\circ} \mathrm{C}$, followed by the opening of second valve to add C3YNE gas. The adding of C3YNE gas was continued until the pressure in the connecting tube rose to 5 bar. Then the second valve was closed, and the first valve was reopened to allow all C3YNE gas to condense in the Parr reactor. This process was repeated for two more times, yielding a total weight of the condensed C3YNE gas equal to $0.33 \pm 0.02 \mathrm{~g}$, as determined by weighing before and after stopping the reaction. The roughly estimated pressure for C3YNE gas in the $10 \mathrm{~cm}^{3}$ reactor was $\sim 13.8 \pm 1$ bar at $25{ }^{\circ} \mathrm{C}$ and $\sim 18.6 \pm 1$ bar at $130^{\circ} \mathrm{C}$. The connection between vessel, C3YNE gas, and argon bottles was then closed, and the vessel was heated to 40$140{ }^{\circ} \mathrm{C}$ for a specified time. The reaction was stopped by allowing the vessel to cool down to room temperature. The remaining C3YNE gas in the vessel was removed by applying vacuum to the system. The setup was then opened under strong argon flow, and the freshly modified surfaces were removed and transported into an argon-purged glovebox for further characterization. [The procedure for 1-butyne was analogous.]

Grignard Reagent Modification of H-Si(111) Surfaces. The twostep chlorination/alkenylation modification ${ }^{23}$ scheme was adapted from the work of Haick et al. ${ }^{24}$ A three-necked glass flask was fitted with a glass capillary through a suitable adapter, a reflux condenser, and a glass stopper. The glass capillary was connected to a Schlenk line to which argon pressure was applied. The reflux condenser was closed with a glass stop which was allowed to hover due to argon flow out of the system. $5 \mathrm{~mL}$ of chlorobenzene was added and bubbled through for $30 \mathrm{~min}$, after which time $\mathrm{PCl}_{5}$ (ca. $50 \mathrm{mg}$ ) was added in small portions using a spatula, until saturation was observed by the precipitation of a white powder, and subsequently a few grains of benzoyl peroxide (ca. $15 \mathrm{mg}$ ) were added. Freshly produced $\mathrm{H}$ terminated $\mathrm{Si}(111)$ substrates were immersed in the chlorination mixture, and the glass was heated to $80{ }^{\circ} \mathrm{C}$ on an oil bath for $1 \mathrm{hr}$. After chlorination, the mixture was left to cool to room temperature. The $\mathrm{Si}$ samples were then thoroughly rinsed with chlorobenzene, anhydrous THF, and anhydrous methanol, dried in an argon jet, and subsequently transported into a glovebox. The chlorinated Si surfaces were (inside the glovebox) put into an oven-dried microwave glass tube to which $1.5 \mathrm{~mL}$ of $0.5 \mathrm{M}$ THF solution of either 1propenylmagnesium bromide, iso-propenylmagnesium bromide, or trans-1-pentenylmagnesium bromide was added. The tube was firmly sealed with a cap using a press and removed from the glovebox. The tube was then placed into an oil bath at $130{ }^{\circ} \mathrm{C}$ and left for $24 \mathrm{~h}$. After cooling of the reaction mixture, the alkenylated surfaces were rinsed with anhydrous THF and then rinsed with anhydrous methanol, a large amount of water, and acetone, followed by 5 min sonication in DCM and a final rinse with DCM. All solvents were removed by drying in an argon jet. The alkenylated $\mathrm{Si}(111)$ surfaces were then stored in a glovebox.

Liquid-Phase 1-Pentyne and 1-Hexyne Modification of H-Si(111) Surfaces. Immediately after preparation, $\mathrm{H}-\mathrm{Si}(111)$ substrates were brought into a glovebox in an oven-dried Schlenk container under vacuum. The surfaces were submerged in $1.5 \mathrm{~mL}$ of 1-pentyne or 1hexyne inside an oven-dried microwave $5 \mathrm{~mL}$ glass tube. Tubes were closed with a Teflon airtight seal using tube crimper. The filled and closed tubes were then removed from the glovebox and placed at room temperature or at $80{ }^{\circ} \mathrm{C}$ in an oil bath for $16 \mathrm{~h}$.

$X$-ray Photoelectron Spectroscopy (XPS). Core electron binding energies were measured by XPS measurements in a JPS-9200 spectrometer (JEOL, Japan). One or two elliptic spots of radius 30 $\mu \mathrm{m}$ were irradiated by a $\mathrm{Al} \mathrm{K} \alpha$ source emitting $1486.7 \mathrm{eV}$ photons, under a voltage of $12 \mathrm{kV}$ through which a current of $20 \mathrm{~mA}$ passed; the analyzer pass energy was $10 \mathrm{eV}$. The high-vacuum chamber had a pressure of $3 \times 10^{-7}$ Torr at $20^{\circ} \mathrm{C}$. A takeoff angle of $80^{\circ}$ was achieved by tilting the sample holder. Spectra were corrected with a slight linear background and subsequently analyzed using CasaXPS version 2.3.16 $\mathrm{PR}$ 1.6. The $\mathrm{C}$ 1s peak emission was calibrated to a binding energy of $285.0 \mathrm{eV}$, and $\mathrm{C}$ 1s narrow scans were deconvoluted into their component peaks using Gaussian-Lorentzian sum functions having 30\%-70\% Gaussian-Lorentzian character. Component peaks were set to a constant fwhm per spectrum.

Quantitative XPS Analysis. For a precise determination of the atomic $\mathrm{C} / \mathrm{Si}$ ratio of organic monolayers on $\mathrm{Si}(111)$, the influence of $\mathrm{X}$-ray photodiffraction (XPD) on the XPS signal has to be accounted for. Therefore, the samples were rotated $120^{\circ}$ around the surface normal (in this orientation the pattern displays a $C_{3}$ symmetry), yielding rotationally averaged $\mathrm{C} 1 \mathrm{~s}$ and $\mathrm{Si} 2 \mathrm{p}$ emissions to obtain a truly quantitative $\mathrm{C} / \mathrm{Si}$ ratio that is independent of the orientation of the sample. As our sample holder only allows rotation of the samples at a takeoff angle of $90^{\circ}$, we used nonmonochromatic $\mathrm{Al} \mathrm{K} \alpha$ X-ray radiation (twin source) at $10 \mathrm{kV}$ and $15 \mathrm{~mA}$ with an analyzer pass energy of $50 \mathrm{eV}$ and a takeoff angle of $90^{\circ}$ for these measurements.

In this work, the monolayer thickness $\left(d_{\mathrm{ML}}, \mathrm{C} / \mathrm{Si}\right)$ was derived from the logarithmic dependence of the $\mathrm{C} 1 \mathrm{~s} / \mathrm{Si} 2 \mathrm{p}$ XPS area intensity ratio (eq 1).

$$
d_{\mathrm{ML}, \mathrm{C} / \mathrm{Si}}=\lambda_{\mathrm{ML}}^{\mathrm{Si}} \sin (\phi) \ln (1+\mathrm{C} / \mathrm{Si})
$$

Here $\lambda_{\mathrm{ML}}^{\mathrm{Si}}$ is the calculated attenuation length of $\mathrm{Si} 2 \mathrm{p}$ photoelectrons (39.5 $\AA$ ), $\phi$ the angle between surface plane and detector $90^{\circ}$, and C/ $\mathrm{Si}$ the atomic ratio of $\mathrm{C} 1 \mathrm{~s}$ and $\mathrm{Si} 2 \mathrm{p}$ peaks as determined from rotationally averaged XPS narrow scans.

Surface Coverage Calculation Method. Throughout literature, surface coverage is often reported by considering $d_{\mathrm{ML}}, \mathrm{C} / \mathrm{Si}$ with reference to alkanethiols on gold. This measure of surface coverage is called $\theta_{\mathrm{ML}, \mathrm{C} / \mathrm{Si}}$ (eq 2). ${ }^{37-40}$ We have also calculated the surface coverages reported in this paper using this method.

$$
\theta_{\mathrm{ML}, \mathrm{C} / \mathrm{Si}}=\frac{d_{\mathrm{ML}, \mathrm{C} / \mathrm{Si}} D_{\mathrm{Au}}}{d_{\mathrm{th}} D_{\mathrm{Si}}}
$$

Here $d_{\mathrm{ML}, \mathrm{C} / \mathrm{Si}}$ is the monolayer thickness as calculated in eq $1, D_{\mathrm{Au}}$ and $D_{\mathrm{Si}}$ are surface site densities for gold and silicon surfaces (respectively $4.65 \times 10^{14}$ and $7.8 \times 10^{14} \mathrm{~cm}^{-2}$ ), and $d_{\mathrm{th}}$ is the predicted monolayer depth from projection of the substituent chain on the surface normal.

Static Water Contact Angle Measurement (SCA). Static water contact angles were measured on a Krüss DSA 100 drop shape analyzer controlled by Krüss DSA software version 1.90.0.14. Three to four drops of $3.0 \mu \mathrm{L}$ of water were deposited on the surface with a microsyringe, and contact angles were determined by fitting the drop shape to the Tangent- 2 model. Each drop was fitted for at least 20 times, and measurements in which left and right contact angle differed more than $1^{\circ}$ were discarded. The error in the resulting data was $\pm 2^{\circ}$.

\section{COMPUTATIONAL METHODS}

Molecular Mechanics Studies of Surface Packing. The dependence of the packing energy $\Delta H$ on the monolayer packing density was studied using molecular mechanics with the Polymer Consistent Force Field (PCFF) under periodic boundary conditions to resemble a crystal surface using the Materials Studio 5.0 package. ${ }^{41}$ A bulk silicon crystal structure was cut along the $\langle 111\rangle$ plane and was placed into a vacuum slab of $3.840 \AA$ wide, $3.840 \AA$ deep. and $10 \AA$ long, so that an initial $\mathrm{Si}_{7}-\mathrm{H}$ unit cell was formed, consisting of four layers of silicon atoms. ${ }^{41}$ The hydrogen on the single Si-atop site was then replaced by the alkene chain forming the monolayer. Covalently bound 1-propenyl, iso-propenyl, 1-butenyl, and isobutenyl chains were studied. Larger unit cells were then constructed by multiplying the initial unit cell along the plane of the surface using the "Supercell" function. Chains were replaced by hydrogen atoms to obtain unit cells with substitution percentages of $25 \%, 33 \%, 50 \%, 60 \%, 67 \%, 75 \%$, 
Scheme 2. (A) Schematic Representation of Gaseous Preparation of C3YNE on $\mathrm{H}-\mathrm{Si}(111)$ Surfaces at $80^{\circ} \mathrm{C}($ iso-C3YNE) and $130{ }^{\circ} \mathrm{C}$ (lin-C3YNE); (B) Two-Step Chlorination and Grignard Alkylation Schemes to Prepare Both iso- (G-iso-C3YNE) and lin- (G-lin-C3YNE) Propenyl Binding Structures

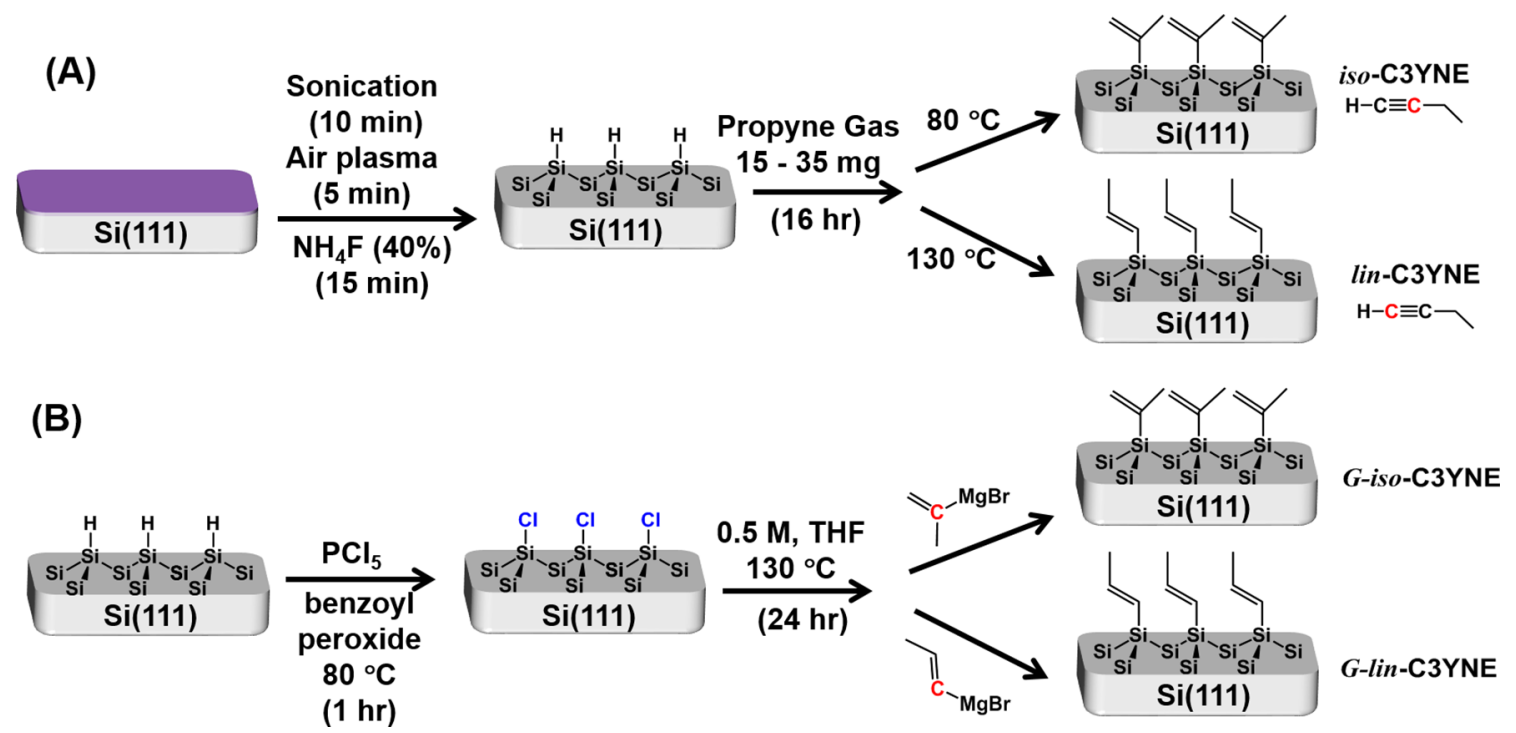

$83 \%$, and $100 \%$. The distinct substitution patterns were used as found in the literature, and several patterns were used per substitution percentage. ${ }^{41-43}$

These unit cells were optimized by the Forcite minimizer, using the PCFF force field, "high-convergence" criteria, and the "smart optimizer" algorithm. Periodic boundary conditions were used to eliminate edge effects. The box dimensions were allowed to be optimized together with the unit cells. To do this correctly, the silicon slab was moved outside of the box; this is necessary as the silicon atoms would otherwise also be optimized, which would not correctly mimic their rigid character. After the optimization, the unit cells were again multiplied in the direction of the plane and reoptimized. This was repeated (2-3 times) until the energy per chain did no longer change. Typical simulation cells consisted of $12 \times 12 \mathrm{Si}$ atop sites.

The $\mathrm{Si}$ atoms were then removed from the model to give only the chains, with radical termination, hanging in a vacuum, the energy of which was obtained in a single-point calculation. The energy of a corresponding free, relaxed alkene radical was then subtracted from the energy of the free, packed system per chain, yielding the energy contribution due to the monolayer packing.

Core Electron Binding Energy Calculations and Total Energy. Geometry optimizations of all molecular species involved in the reaction were performed at the B3LYP/6$311 G(d, p)$ level of theory. Electronic core level binding energies were calculated by performing natural bond orbital $(\mathrm{NBO})^{44}$ analysis on fully optimized geometries at the same level of theory, and the thus obtained core binding energies were later used to simulate the C 1s XPS spectra. ${ }^{33}$ All calculations were carried out by using the Gaussian09 (revision D1) quantum software package. ${ }^{33,45}$ The surface-monolayer system was mimicked by binding an alkenyl chain to a trimethylsilane $\left(-\mathrm{Si}\left(\mathrm{CH}_{3}\right)_{3}\right)$ moiety, as used before to mimic the Si surface. ${ }^{35,40}$ Total energy was calculated via high-quality $\mathrm{G} 3 \mathrm{ab}$ initio calculations of the reaction of C3YNE and C4YNE both with iso as well as lin with a small hydrogen-terminated silicon cluster $\left(\mathrm{HSi}\left(\mathrm{SiH}_{3}\right)_{3}\right)$, analogous to literature procedures performed using the Gaussian 09 package.

\section{RESULTS AND DISCUSSION}

Gas-Phase Deposition of Monolayers. To assess the use of gas-phase alkynes for the formation of covalent monolayers on $\mathrm{H}-\mathrm{Si}(111)$, a home-built Parr-vesse-based reactor was assembled as drawn in Scheme 1. Fresh $\mathrm{H}-\mathrm{Si}(111)$ chips were placed in the reactor, and the alkynes (propyne, 1-butyne, 1pentyne, or 1-hexyne) were subsequently condensed in there. Next, the reaction vessel was heated to allow covalent bond formation as shown in Scheme 2A for the case of C3YNE. The surface attachment was studied using different temperatures and gas amounts in order to optimize the reaction. The resulting surfaces were analyzed by XPS and SCA to infer the structure of the organic layer. The XPS and SCA results are summarized in Table 1. The C 1s XPS narrow scans and DFTcalculated C 1s XPS spectra are shown in Figure 1.

Figure 1A shows the $\mathrm{C} 1 \mathrm{~s}$ narrow scan XPS data of $\mathrm{H}$ terminated $\mathrm{Si}(111)$ surfaces modified with $\mathrm{C} 3 \mathrm{YNE}$ gas at 80 ${ }^{\circ} \mathrm{C}$. The spectrum is remarkable in the absence of any shoulder peaks: only a peak at $285.0 \mathrm{eV}$ is visible. $\mathrm{Si}-\mathrm{C}$ bound alkenyl monolayers typically show a shoulder at $283.9 \mathrm{eV}$ that is attributed to the first carbon atom in the linear chain $\mathrm{Si}-\mathrm{C}=$ C. ${ }^{37}$ The shorter the alkyne that is reacted to the surface, the easier it is to detect this shoulder, so if the characteristic peak is not visible even in chains as short as derived from C3YNE, it is likely simply not there. Therefore, this result was interpreted to indicate that $\mathrm{Si}-\mathrm{C}$ binding of C3YNE occurs through another, unprecedented attachment chemistry than observed for the surface hydrosilylation of any alkene or alkyne. Interestingly, upon increase of the reaction temperature to $130{ }^{\circ} \mathrm{C}$, this characteristic and clearly distinct peak at $283.9 \mathrm{eV}$ does become visible, as shown in Figure 1D (lin-C3YNE). This indicates that at these elevated temperatures the binding occurs through the C1 carbon of C3YNE. The shoulder peak at $283.9 \mathrm{eV}$ was absent in all experiments below $90{ }^{\circ} \mathrm{C}$ and observed for all reaction temperatures above that threshold value (see Table 1 ). 
Table 1. Modification of $\mathrm{H}-\mathrm{Si}(111)$ Surfaces by C3YNE at Elevated Pressure and Various Temperatures: Summary of Ellipsometry, XPS, and Water Static Contact Angle (SCA) Data

\begin{tabular}{|c|c|c|c|c|c|c|}
\hline \multirow[b]{2}{*}{$\begin{array}{l}\text { temp } \\
\left({ }^{\circ} \mathrm{C}\right)\end{array}$} & \multirow[b]{2}{*}{$\begin{array}{l}d_{\text {ellips }} \\
(\AA)\end{array}$} & \multirow[b]{2}{*}{$\underset{(\operatorname{deg})^{b}}{\text { SCA }}$} & \multicolumn{4}{|c|}{ XPS data and coverage } \\
\hline & & & C $1 \mathrm{~s} / \mathrm{Si} 2 \mathrm{p}^{c}$ & $\begin{array}{l}d_{\mathrm{ML}} \\
(\tilde{A})\end{array}$ & $\begin{array}{l}\theta_{\mathrm{ML}} \\
(\%)\end{array}$ & $=\mathrm{C}-\mathrm{Si} / \mathrm{Si} 2 \mathrm{p}^{d}$ \\
\hline 25 & 3.8 & 74 & $7.8 / 92.2$ & 3.2 & 44 & $-{ }^{e}$ \\
\hline 40 & 3.8 & 77 & $10.3 / 89.7$ & 4.2 & 59 & $-{ }^{e}$ \\
\hline 60 & 4.5 & 80 & $12.8 / 87.1$ & 5.4 & 75 & $-^{e}$ \\
\hline 80 & 5.8 & 85 & $13.9 / 86.1$ & 5.8 & 81 & $-^{e}$ \\
\hline 90 & 5.7 & 88 & $14.2 / 85.8$ & 6.0 & 83 & $2.8 / 85.1$ \\
\hline 110 & 6.3 & 89 & $14.8 / 85.2$ & 6.2 & 87 & $4.4 / 85.6$ \\
\hline 120 & 7.5 & 89 & $15.1 / 84.9$ & 6.4 & 89 & $4.9 / 85.1$ \\
\hline 130 & 6.9 & 90 & $15.5 / 84.5$ & 6.6 & 92 & $5.5 / 83.7$ \\
\hline
\end{tabular}

${ }^{a}$ Ellipsometry thickness $( \pm 1 \AA) .{ }^{b}$ Static water contact angle $\left( \pm 1^{\circ}\right)$. ${ }^{c} \mathrm{C} / \mathrm{Si}$ ratio from rotationally averaged XPS spectra. ${ }^{d}$ Calculated from narrow scan of $\mathrm{C} 1 \mathrm{~s}$ fitted to two peaks $(\mathrm{C}-\mathrm{C}(285.0 \mathrm{eV})$ and $=\mathrm{C}-\mathrm{Si}$ $(283.9 \mathrm{eV}))$ and Si $2 \mathrm{p}(99.9 \mathrm{eV})$ area $\%$. ${ }^{e}$ At these temperatures no $=\mathrm{C}-\mathrm{Si}$ shoulder peak at $283.9 \mathrm{eV}$ was observed in the $\mathrm{C} 1 \mathrm{~s}$ narrow scan.

Several options were considered for the low-temperature structure, including 1,2-cyclized C3YNE products and fully saturated monolayers. However, the first would be high in energy, and perhaps also reactive itself, whereas the second is improbable given the reaction conditions. The C 1s XPS spectra for a variety of binding options were simulated by DFT quantum chemical methods that previously showed reliable results. ${ }^{33,46}$ These simulated C 1s XPS spectra indicated that the XPS data could be explained by assuming the lowtemperature reactivity of C3YNE to take place at the 2-position and at the (regularly observed for hydrosilylation reactions) 1- position at higher temperatures. The $\mathrm{C} 1 \mathrm{~s}$ binding energies for $\mathrm{Si}-\underline{C}=$ binding at the iso position (iso-C3YNE; lower temperature) and at the terminal position (lin-C3YNE, higher temperature) are calculated to be 285.0 and $284.3 \mathrm{eV}$, respectively. These data are in reasonable agreement with the experimental C 1s XPS results (Figure 1) of 285.0 and 283.9 $\mathrm{eV}$, respectively.

To confirm this, the two-step chlorination and Grignard alkylation route using iso-propenylmagnesium bromide and 1propenylmagnesium bromide (Scheme 2B) was employed to produce the G-iso-C3YNE and G-lin-C3YNE monolayers, respectively, in a well-defined manner following previously reported methods. ${ }^{3,23-25,31,47-49}$ The XPS C 1s narrow scans are presented in Figures $1 \mathrm{~B}$ and $1 \mathrm{E}$, respectively. Both experimental (via the one-step, gas-phase reaction and the two-step Grignard alkenylation) and simulated XPS spectra show that the branched propenyl monolayer gives a single, narrow carbon peak that resists fitting with an additional $=\mathrm{C}-\mathrm{Si}$ component at $283.9 \mathrm{eV}$ that is observed for linearly bound propenyl chains.

The detailed quantification data of the various temperature effects on the modification of $\mathrm{H}-\mathrm{Si}(111)$ surfaces using a constant amount of C3YNE $(330 \pm 20 \mathrm{mg})$ and fixed reaction time $(16 \mathrm{~h})$ are shown in Table 1 . The static water contact angles (SCA) of the C3YNE-derived monolayers on $\mathrm{H}-$ $\mathrm{Si}(111)$ monolayers increase with increasing temperature. The SCAs for modified monolayers at reaction temperatures lower than $90^{\circ} \mathrm{C}$ were found be between $74^{\circ}$ and $85 \pm 1^{\circ}$, i.e., lower than freshly etched $\mathrm{H}-\mathrm{Si}(111)$ surfaces $\left(86^{\circ}\right)$. In contrast, the SCAs for C3YNE-modified monolayers at reaction temperatures higher than $90^{\circ} \mathrm{C}$ were found to be $90 \pm 1^{\circ}$, as shown in Table 1. These data can be interpreted, given the difference in typical SCA of long-chain $\left(>\mathrm{C}_{10}\right) \mathrm{CH}_{3}$-terminated monolayers $\left(\sim 110^{\circ}-111^{\circ}\right)^{38,39}$ with that of long-chain alkene-terminated
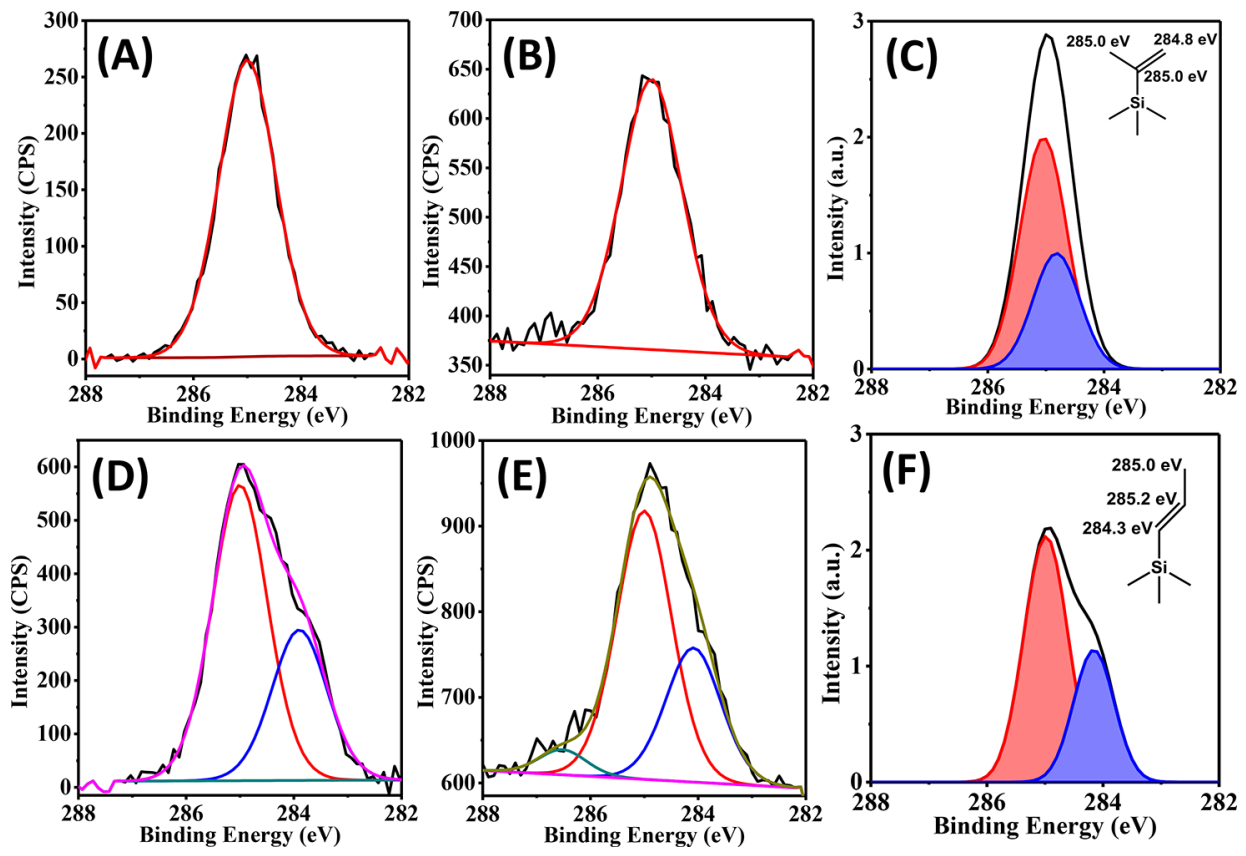

Figure 1. XPS narrow scan for the $\mathrm{C} 1 \mathrm{~s}$ region of propynyl-modified $\mathrm{H}-\mathrm{Si}(111)$ surfaces. (A) Substrate from gas-phase modification with C3YNE at $80{ }^{\circ} \mathrm{C}$, iso-C3YNE. (B) Substrate resulting from Grignard alkenylation using iso-propenylmagnesium bromide at $130{ }^{\circ} \mathrm{C}, \mathrm{G}$-iso-C3YNE. (C) DFTcalculated XPS spectra for iso-C3YNE. (D) Substrate from gas-phase modification with C3YNE at $130{ }^{\circ} \mathrm{C}$, lin-C3YNE. (E) Substrate resulting from Grignard alkenylation using 1-propenylmagnesium bromide at $130{ }^{\circ} \mathrm{C}$, G-lin-C3YNE. (F) DFT-calculated C 1s XPS spectra for lin-C3YNE. The fwhm in the simulations was set to the constant fwhm of the component peaks fitted to the measured XPS scans. 

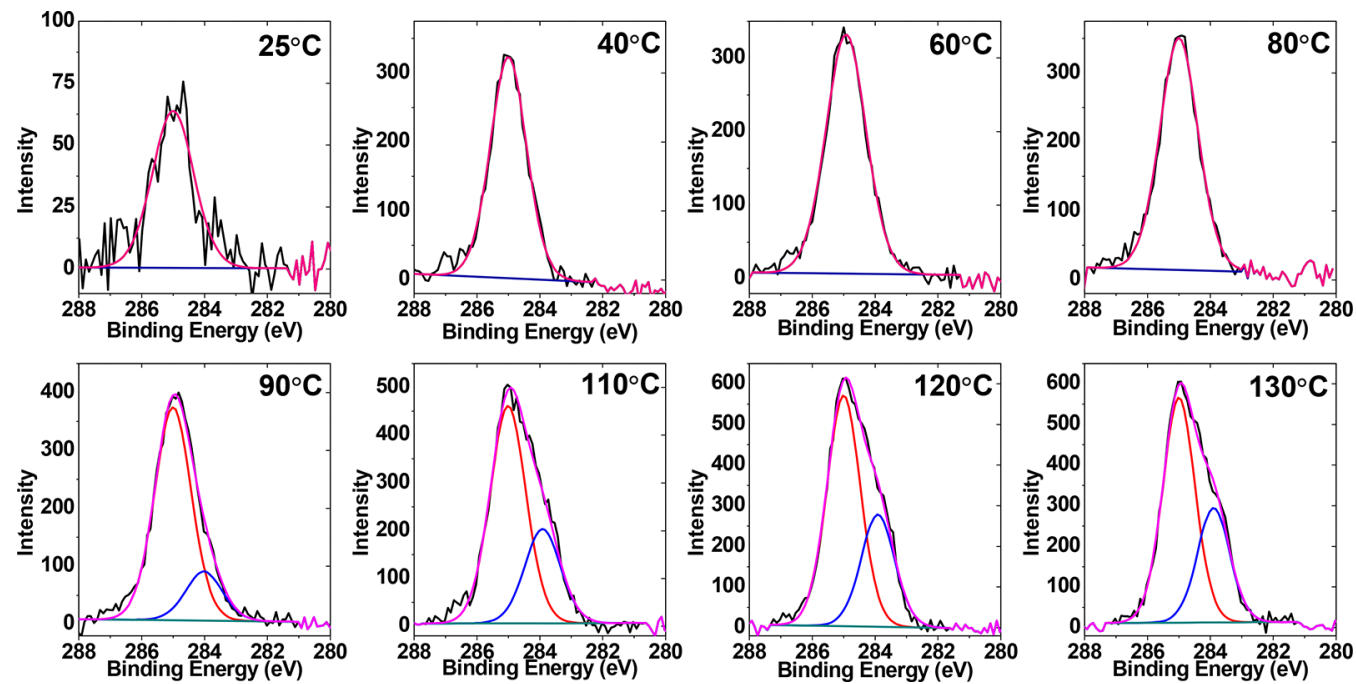

Figure 2. Narrow scan $\mathrm{C} 1 \mathrm{~s}$ spectra of $\mathrm{C} 3 \mathrm{YNE}$-derived monolayers on $\mathrm{H}-\mathrm{Si}(111)$ surfaces obtained at reaction temperatures from 25 to $130{ }^{\circ} \mathrm{C}$.

Table 2. Surface Modification of $\mathrm{H}-\mathrm{Si}(111)$ Surfaces by High-Pressure Gas-Phase Hydrosilylation with 1-Alkynes (Only linC3YNE Was Prepared at $130{ }^{\circ} \mathrm{C}$ While All Other Monolayers Were Prepared at $80{ }^{\circ} \mathrm{C}, 16 \mathrm{~h}$ ) and Grignard Reaction with Analogous Alkenylmagnesium Bromides: Ellipsometry, Static Water Contact Angle (SCA), and XPS-Derived Data

\begin{tabular}{lccccccc} 
& & & & \multicolumn{4}{c}{ XPS data and coverage } \\
\cline { 6 - 8 } monolayers & $d_{\text {ellips }}(\AA)^{a}$ & $\mathrm{SCA}(\mathrm{deg})^{b}$ & $\mathrm{C} 1 \mathrm{~s} / \mathrm{Si} 2 \mathrm{p}^{c}$ & $d_{\mathrm{ML}}(\AA)$ & $\theta_{\mathrm{ML}}(\%)$ & $=\mathrm{C}-\mathrm{Si} / \mathrm{Si} 2 \mathrm{p}^{d}$ & $=\mathrm{C}-\mathrm{Si} / \mathrm{C}-\mathrm{C}$ \\
iso-C3YNE & 5.8 & 85 & $13.9 / 86.1$ & 5.8 & 81 & $-^{e}$ \\
lin-C3YNE & 6.9 & 90 & $15.5 / 84.5$ & 6.6 & 92 & $5.51 / 83.7$ & $33.8 / 66.2$ \\
G-iso-C3YNE & 3.4 & 85 & $10.2 / 89.8$ & 4.2 & 58 & $-{ }^{e}$ & \\
G-lin-C3YNE & 5.0 & 88 & $13.3 / 86.6$ & 5.6 & 78 & $4.76 / 85.7$ & $38.6 / 61.4$ \\
C4YNE & 7.0 & 90 & $17.3 / 82.7$ & 7.9 & 88 & $4.78 / 80.7$ & $24.8 / 75.2$ \\
C5YNE & 7.0 & 98 & $20.1 / 79.9$ & 8.7 & 77 & $3.66 / 81.7$ & $20.0 / 80.0$ \\
C6YNE & 8.0 & 103 & $21.4 / 78.6$ & 9.4 & 71 & $3.37 / 79.8$ & $16.7 / 83.3$
\end{tabular}

${ }^{a}$ Ellipsometry thickness $( \pm 1 \AA) .{ }^{b}$ Static water contact angle $\left( \pm 1^{\circ}\right) .{ }^{c} \mathrm{C} / \mathrm{Si}$ ratio from rotationally averaged XPS spectra. ${ }^{d}$ Calculated from narrow scan of $\mathrm{C} 1 \mathrm{~s}$ fitted to two peaks $(\mathrm{C}-\mathrm{C}(285.0 \mathrm{eV})$ and $=\mathrm{C}-\mathrm{Si}(283.9 \mathrm{eV}))$ and $\mathrm{Si} 2 \mathrm{p}(99.9 \mathrm{eV})$ area $\%{ }^{e}$ At these condition no $=\mathrm{C}-\mathrm{Si}$ shoulder peak at $283.9 \mathrm{eV}$ was observed in the $\mathrm{C} 1 \mathrm{~s}$ narrow scan.

$\left(\mathrm{CH}=\mathrm{CH}_{2}\right)$ monolayers $\left(\sim 96^{\circ}\right) .{ }^{50}$ The lower SCA values observed here at lower reaction temperatures are again consistent with reaction in a branched iso fashion at lower temperatures $\left(<80{ }^{\circ} \mathrm{C}\right)$ and in lin fashion at higher temperatures $\left(>90{ }^{\circ} \mathrm{C}\right)$. This is further substantiated by the ellipsometric thickness that shows an increase from 3.8 to 6.9 $\AA$ for the temperature range from 25 to $130{ }^{\circ} \mathrm{C}$. As found more often, ${ }^{38}$ the ellipsometric thicknesses are slightly overestimated as compared to the theoretical thickness for linearly bound C3YNE, which is calculated to $4.4 \AA$.

Further, we have used the rotationally averaged XPS method of Wallart and co-workers ${ }^{51}$ to calculate the surface coverages of C3YNE-derived monolayers, and also these data are shown in Table 1. This approach allows to calculate the surface coverages by using the $\mathrm{C} 1 \mathrm{~s} / \mathrm{Si} 2 \mathrm{p}$ ratio at different rotation azimuth angles from $0^{\circ}$ to $120^{\circ}$ (eqs 1 and 2). ${ }^{51}$ The C3YNE monolayers thickness were found to be between 3.2 and $6.6 \AA$, and the surface coverages range from $44 \%$ to $92 \%$ with reaction temperatures from 25 to $130{ }^{\circ} \mathrm{C}$. The $\mathrm{C} 1$ s peak of $=\underline{C}-\mathrm{Si}$ at $283.9 \mathrm{eV}$ only starts to observed from $90{ }^{\circ} \mathrm{C}$ onward. A further increased intensity is found for increasing temperatures as shown in Figure 2, showing a more densely packed monolayer. At the highest temperatures $\left(120\right.$ and $\left.130{ }^{\circ} \mathrm{C}\right)$, the $\underline{C}-\mathrm{C} /=\underline{C}-$ $\mathrm{Si}$ ratio has reached the theoretical ratio of $2: 1$. This indicates the formation of a monolayer in the absence of multilayer formation, such as observed in e.g. the photochemical surface attachment of alkynes onto $\mathrm{H}-\mathrm{Si}(111)$ using $254 \mathrm{~nm}$ light. In addition, this gas-phase method did not yield any $\mathrm{C}-\mathrm{O}$ peak at $286.7 \mathrm{eV}$, indicative of atmospheric contamination, which was observed as a little peak in the literature ${ }^{48}$ and in our experiments for various monolayers obtained via the Grignard method. $^{24,25,29,47-49}$

Furthermore, the surface coverages obtained from this novel method (ranging from $44 \%$ to $92 \%$ with reaction temperatures from 25 to $130{ }^{\circ} \mathrm{C}$ ) are higher than those we obtained using the Grignard method-58\% for G-iso-C3YNE and 78\% for G-linC3YNE monolayers-as shown in Table 2, whereas our Grignard data compare well with regard to literature data (reporting e.g. $56 \%$ for 1-propenyl-terminated monolayers). ${ }^{24,29,48,49}$ In conclusion, these results indicate that monolayers prepared by the one-step gas-phase hydrosilylation process are not only obtained without involvement of harsh chemicals but are also significantly more dense than analogous monolayers prepared by a Grignard reaction.

Chain Length Effects. The quality of short chain length $\left(\mathrm{C}_{3}-\mathrm{C}_{6}\right)$ monolayers on $\mathrm{H}-\mathrm{Si}(111)$ surfaces modified under high pressure and using a reaction time and temperature often used for the attachment of longer 1-alkynes $\left(16 \mathrm{~h}\right.$ at $\left.80{ }^{\circ} \mathrm{C}\right)$ is presented in Table 2. The iso-C3YNE and lin-C3YNE monolayers were obtained at 80 and $130{ }^{\circ} \mathrm{C}$. C4YNE 
monolayers were also prepared at various temperatures from 25 to $130{ }^{\circ} \mathrm{C}$. At all these temperatures, it was found that C4YNE attachment exclusively happened in linear fashion (see Supporting Information, Figure S1). Surface coverages were calculated for C4YNE monolayers and were found to be $88 \pm$ $4 \%$, with a thickness of $7.9 \AA$ at all investigated temperatures. Analogous monolayers derived from 1-pentyne and 1-hexyne yielded surface coverages of $77 \%$ and $71 \%$, respectively. The $C$ 1 s narrow spectrum for different chain length revealed a $=\mathrm{C}-$ $\mathrm{Si} / \mathrm{C}-\mathrm{C}$ ratio, which agrees well with the expected theoretical value of 33/66 for C3YNE, 25/75 for C4YNE, 20/80 for C5YNE, and 16.6/83.3 C6YNE as shown in Table 2. These results indicate that the surface coverage decreases with increasing the chain length: C3YNE > C4YNE > C5YNE > C6YNE: with increasing steric bulk, the optimized packing density becomes lower.

Packing and Binding Energies. The packing of $\mathrm{C}_{3}$ and $\mathrm{C}_{4}$ alkenyl-derived $\mathrm{Si}(111)$ monolayers was investigated by molecular mechanics studies. Unit cells consisting of repeating $\mathrm{Si}_{7}$ units (four layers) were constructed, and top sites were bonded to either hydrogen or an alkenyl chain. Four systems were modeled-iso-C3YNE, iso-C4YNE, lin-C3YNE, and linC4YNE- to see how the mode of attachment and chain length impact the order of the resulting monolayer.

To study the interchain interactions, the packing energy per chain is calculated; see Figure 3 (open symbols). The packing
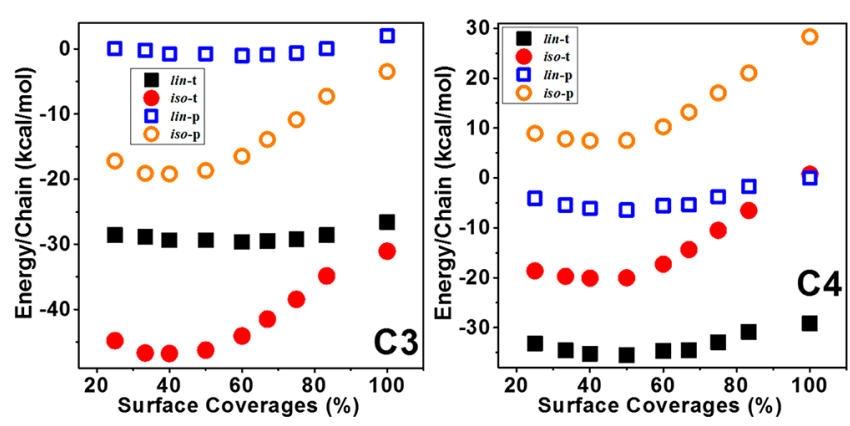

Figure 3. Packing energies ("p", open) and total energies ("t", solid) per binding molecule as a function of the surface coverage for both C3YNE (left) and C4YNE (right) monolayers.

energy is the energy that is consumed or released by adding a chain, close to another chain. The packing energy can be calculated with the equation ${ }^{41}$

$$
E_{\text {packing }}=\frac{E_{\text {chains }}}{n}-E_{\text {relaxed chain }}
$$

where $E_{\text {chains }}$ is the total packing energy of all chains in an optimized simulation cell, $n$ the total number of chains in the same unit cell, and $E_{\text {relaxed chain }}$ the potential energy of a single free optimized alkene, which represents an attached alkyne chain. To obtain the packing energy of the chains, the silicon layer has to be cut off; i.e., all $\mathrm{Si}-\mathrm{C}$ bonds were cleaved.

However, the relative energies of the monolayers resulting from iso and lin attachment are not determined by packing alone, as the free energy of $\mathrm{Si}-\mathrm{C}$ bond formation $\left(\Delta G_{\text {binding }}\right)$ is different for these two attachment modes. Using high-level G3 calculations on an $\left(\mathrm{SiH}_{3}\right)_{3} \mathrm{Si}-\mathrm{H}$ cluster, $\Delta G_{\text {binding }}$ was therefore determined for both lin and iso products. The packing energy (eq 3) and total (packing + binding; eq 4) energy as a function of $\mathrm{H}-\mathrm{Si}$ substitution fraction are given in Figure 3.

The total energy/chain is calculated by using the equation

$$
\begin{aligned}
E_{\text {total }} & =\Delta G_{\text {bonding }}+E_{\text {packing }} \\
& =\Delta G_{\text {bonding }}+\frac{E_{\text {chains }}}{n}-E_{\text {relaxed chain }}
\end{aligned}
$$

where $\Delta G_{\text {bonding }}$ is assumed to be independent of packing density.

The data in Figure 3 reveal three trends: (1) For C3YNE the packing energy for iso attachment for low to medium monolayer packing densities is much lower than for lin attachment. Given the higher steric hindrance of iso-attached propenyl chains, their optimum packing density will also be lower, as in indeed calculated and observed. In line with this, with increasing packing density the packing energy of iso monolayers goes up much faster than that of lin monolayers. (2) The G3-calculated binding energy for iso attachment is $-27.6 \mathrm{kcal} / \mathrm{mol}$, whereas for lin attachment this is $-28.6 \mathrm{kcal} /$ mol. ${ }^{30,52}$ The more stable linear binding mode causes a crossover of monolayer stabilities for C3YNE at higher packing densities: at low densities, iso is more stable; at higher densities the lin attachment wins out. (3) For longer chains ( $C_{4}$ and up), the added sterics of iso attachment cause the linear attachment to be preferred at all packing densities, explaining why linear attachment has been the only mode of attachment ever observed up to now for long-chain alkynes. In addition, the optimum packing density for both the iso and lin monolayers are lower than calculated for C3YNE-derived monolayers, again in line with experiment.

While these data point to the factors that govern the mode of attachment, they do not yet explain the details of why iso attachment takes place at low temperatures but is taken over by lin attachment at higher temperatures. More detailed experiments and quantum chemical calculations to study this are currently in progress, together with the further development and exploitation of this technique for use on other surfaces.

\section{CONCLUSIONS}

A novel catalyst-free gas-phase hydrosilylation of H-terminated $\mathrm{Si}(111)$ surfaces was developed, which allows for the smooth attachment of ordered, high-density monolayers of low-boiling materials, such as propyne and 1-butyne. Detailed surface characterization combined with molecular modeling shows that propyne binds at low temperatures via an as of yet unprecedented iso mode of attachment, forming a $\mathrm{Si}-$ $\mathrm{C}\left(\mathrm{CH}_{3}\right)=\mathrm{CH}_{2}$ structure. Only at increased temperatures or with increasing the size of the molecule (1-butyne and larger alkynes) does a crossover to the standard linear mode of attachment occur. Given the high surface coverages that can be obtained in a solvent-free manner (up to $92 \%$ for propyne), this approach also points to novel, highly scalable routes to the surface modification of a wide range of materials, including semiconductors and $\mathrm{Si}-\mathrm{H}$ modified oxides. ${ }^{53}$

\section{ASSOCIATED CONTENT}

\section{Supporting Information}

The Supporting Information is available free of charge on the ACS Publications website at DOI: 10.1021/acs.langmuir.7b03683.

Figures S1 and S2 (PDF)

\section{AUTHOR INFORMATION}

\section{Corresponding Author}

*Phone+31-317-482361; e-mail Han.Zuilhof@wur.nl (H.Z.). 


\section{ORCID}

Sidharam P. Pujari: 0000-0003-0479-8884

Han Zuilhof: 0000-0001-5773-8506

Notes

The authors declare no competing financial interest.

\section{ACKNOWLEDGMENTS}

This work was supported by NanoNextNL, a micro and nanotechnology consortium of the Government of The Netherlands and 130 partners (program 6C, Beyond Moore)).

\section{REFERENCES}

(1) Linford, M. R.; Chidsey, C. E. D. Alkyl Monolayers Covalently Bonded to Silicon Surfaces. J. Am. Chem. Soc. 1993, 115 (26), 1263112632.

(2) Li, Y.; Calder, S.; Yaffe, O.; Cahen, D.; Haick, H.; Kronik, L.; Zuilhof, H. Hybrids of Organic Molecules and Flat, Oxide-Free Silicon: High-Density Monolayers, Electronic Properties, and Functionalization. Langmuir 2012, 28 (26), 9920-9929.

(3) Wong, K. T.; Lewis, N. S. What a Difference a Bond Makes: The Structural, Chemical, and Physical Properties of Methyl-Terminated Si(111) Surfaces. Acc. Chem. Res. 2014, 47 (10), 3037-3044.

(4) DeBenedetti, W. J. I.; Chabal, Y. J. Functionalization of oxide-free silicon surfaces. J. Vac. Sci. Technol., A 2013, 31 (5), 050826.

(5) Tao, F.; Bernasek, S. L. Functionalization of Semiconductor Surfaces; John Wiley \& Sons, Inc.: 2012.

(6) Fabre, B. Functionalization of Oxide-Free Silicon Surfaces with Redox-Active Assemblies. Chem. Rev. 2016, 116 (8), 4808-4849.

(7) Seitz, O.; Böcking, T.; Salomon, A.; Gooding, J. J.; Cahen, D. Importance of Monolayer Quality for Interpreting Current Transport through Organic Molecules: Alkyls on Oxide-Free Si. Langmuir 2006, 22 (16), 6915-6922.

(8) Koleini, M.; Colombi Ciacchi, L.; Fernández-Serra, M. V. Electronic Transport in Natively Oxidized Silicon Nanowires. ACS Nano 2011, 5 (4), 2839-2846.

(9) Teplyakov, A. V.; Bent, S. F. Semiconductor Surface Functionalization for Advances in Electronics, Energy Conversion, and Dynamic Systems. J. Vac. Sci. Technol., A 2013, 31 (5), 050810.

(10) Vilan, A.; Yaffe, O.; Biller, A.; Salomon, A.; Kahn, A.; Cahen, D. Molecules on Si: Electronics with Chemistry. Adv. Mater. 2010, 22 (2), $140-159$.

(11) Wang, J.; Shen, F.; Wang, Z.; He, G.; Qin, J.; Cheng, N.; Yao, M.; Li, L.; Guo, X. Point Decoration of Silicon Nanowires: An Approach Toward Single-Molecule Electrical Detection. Angew. Chem., Int. Ed. 2014, 53 (20), 5038-5043.

(12) Strother, T.; Cai, W.; Zhao, X.; Hamers, R. J.; Smith, L. M. Synthesis and Characterization of DNA-Modified Silicon (111) Surfaces. J. Am. Chem. Soc. 2000, 122 (6), 1205-1209.

(13) Pike, A. R.; Lie, L. H.; Eagling, R. A.; Ryder, L. C.; Patole, S. N.; Connolly, B. A.; Horrocks, B. R.; Houlton, A. DNA On Silicon Devices: On-Chip Synthesis, Hybridization, and Charge Transfer. Angew. Chem., Int. Ed. 2002, 41 (4), 615-617.

(14) Fabre, B.; Li, Y.; Scheres, L.; Pujari, S. P.; Zuilhof, H. LightActivated Electroactive Molecule-Based Memory Microcells Confined on a Silicon Surface. Angew. Chem. 2013, 125 (46), 12246-12249.

(15) Masuda, T.; Fukumitsu, H.; Takakusagi, S.; Chun, W.-J.; Kondo, T.; Asakura, K.; Uosaki, K. Molecular Catalysts Confined on and Within Molecular Layers Formed on a Si(111) Surface with Direct SiC Bonds. Adv. Mater. 2012, 24 (2), 268-272.

(16) Sieval, A. B.; Demirel, A. L.; Nissink, J. W. M.; Linford, M. R.; van der Maas, J. H.; de Jeu, W. H.; Zuilhof, H.; Sudhölter, E. J. R. Highly Stable Si-C Linked Functionalized Monolayers on the Silicon (100) Surface. Langmuir 1998, 14 (7), 1759-1768.

(17) Sun, Q.-Y.; de Smet, L. C. P. M.; van Lagen, B.; Giesbers, M.; Thüne, P. C.; van Engelenburg, J.; de Wolf, F. A.; Zuilhof, H.; Sudhölter, E. J. R. Covalently Attached Monolayers on Crystalline
Hydrogen-Terminated Silicon: Extremely Mild Attachment by Visible Light. J. Am. Chem. Soc. 2005, 127 (8), 2514-2523.

(18) Stewart, M. P.; Buriak, J. M. Exciton-Mediated Hydrosilylation on Photoluminescent Nanocrystalline Silicon. J. Am. Chem. Soc. 2001, 123 (32), 7821-7830.

(19) Huck, L. A.; Buriak, J. M. UV-Initiated Hydrosilylation on Hydrogen-Terminated Silicon (111): Rate Coefficient Increase of Two Orders of Magnitude in the Presence of Aromatic Electron Acceptors. Langmuir 2012, 28 (47), 16285-16293.

(20) Eves, B. J.; Lopinski, G. P. Formation of Organic Monolayers on Silicon via Gas-Phase Photochemical Reactions. Langmuir 2006, 22 (7), 3180-3185.

(21) Stewart, M. P.; Robins, E. G.; Geders, T. W.; Allen, M. J.; Cheul Choi, H.; Buriak, J. M. Three Methods for Stabilization and Functionalization of Porous Silicon Surfaces via Hydrosilylation and Electrografting Reactions. Phys. Status Solidi (a) 2000, 182 (1), 109115 .

(22) Linford, M. R.; Fenter, P.; Eisenberger, P. M.; Chidsey, C. E. D. Alkyl Monolayers on Silicon Prepared from 1-Alkenes and HydrogenTerminated Silicon. J. Am. Chem. Soc. 1995, 117 (11), 3145-3155.

(23) Bansal, A.; Li, X.; Lauermann, I.; Lewis, N. S.; Yi, S. I.; Weinberg, W. H. Alkylation of Si Surfaces Using a Two-Step Halogenation/Grignard Route. J. Am. Chem. Soc. 1996, 118 (30), $7225-7226$.

(24) Puniredd, S. R.; Assad, O.; Haick, H. Highly Stable Organic Monolayers for Reacting Silicon with Further Functionalities: The Effect of the C-C Bond nearest the Silicon Surface. J. Am. Chem. Soc. 2008, 130 (41), 13727-13734.

(25) Hurley, P. T.; Nemanick, E. J.; Brunschwig, B. S.; Lewis, N. S. Covalent Attachment of Acetylene and Methylacetylene Functionality to $\mathrm{Si}(111)$ Surfaces: Scaffolds for Organic Surface Functionalization while Retaining $\mathrm{Si}-\mathrm{C}$ Passivation of $\mathrm{Si}(111)$ Surface Sites. J. Am. Chem. Soc. 2006, 128 (31), 9990-9991.

(26) Plymale, N. T.; Kim, Y.-G.; Soriaga, M. P.; Brunschwig, B. S.; Lewis, N. S. Synthesis, Characterization, and Reactivity of Ethynyl- and Propynyl-Terminated Si(111) Surfaces. J. Phys. Chem. C 2015, 119 (34), 19847-19862.

(27) de Villeneuve, C. H.; Pinson, J.; Bernard, M. C.; Allongue, P. Electrochemical Formation of Close-Packed Phenyl Layers on $\mathrm{Si}(111)$. J. Phys. Chem. B 1997, 101 (14), 2415-2420.

(28) Bhairamadgi, N. S.; Pujari, S. P.; Trovela, F. G.; Debrassi, A.; Khamis, A. A.; Alonso, J. M.; Al Zahrani, A. A.; Wennekes, T.; AlTuraif, H. A.; van Rijn, C.; Alhamed, Y. A.; Zuilhof, H. Hydrolytic and Thermal Stability of Organic Monolayers on Various Inorganic Substrates. Langmuir 2014, 30 (20), 5829-5839.

(29) Bashouti, M. Y.; Garzuzi, C. A.; de la Mata, M.; Arbiol, J.; Ristein, J.; Haick, H.; Christiansen, S. Role of Silicon Nanowire Diameter for Alkyl (Chain Lengths C1-C18) Passivation Efficiency through Si-C Bonds. Langmuir 2015, 31 (8), 2430-2437.

(30) Soria, F. A.; Paredes-Olivera, P.; Patrito, E. M. Chemical Stability toward $\mathrm{O} 2$ and $\mathrm{H}_{2} \mathrm{O}$ of $\mathrm{Si}(111)$ Grafted with $-\mathrm{CH} 3,-$ $\mathrm{CH} 2 \mathrm{CH} 2 \mathrm{CH} 3,-\mathrm{CHCHCH} 3$, and $-\mathrm{CCCH} 3$. J. Phys. Chem. C 2015, 119 (1), 284-295.

(31) Bashouti, M. Y.; Sardashti, K.; Schmitt, S. W.; Pietsch, M.; Ristein, J.; Haick, H.; Christiansen, S. H. Oxide-free hybrid silicon nanowires: From fundamentals to applied nanotechnology. Prog. Surf. Sci. 2013, 88 (1), 39-60.

(32) Sen, R.; Escorihuela, J.; van Delft, F.; Zuilhof, H. Rapid and Complete Surface Modification with Strain-Promoted OxidationControlled Cyclooctyne-1,2-Quinone Cycloaddition (SPOCQ). Angew. Chem. 2017, 129 (12), 3347-3351.

(33) Giesbers, M.; Marcelis, A. T. M.; Zuilhof, H. Simulation of XPS C1s Spectra of Organic Monolayers by Quantum Chemical Methods. Langmuir 2013, 29 (15), 4782-4788.

(34) Zhao, J.; Gao, F.; Pujari, S. P.; Zuilhof, H.; Teplyakov, A. V. Universal Calibration of Computationally Predicted N 1s Binding Energies for Interpretation of XPS Experimental Measurements. Langmuir 2017, 33, 10792. 
(35) Rijksen, B.; van Lagen, B.; Zuilhof, H. Mimicking the Silicon Surface: Reactivity of Silyl Radical Cations toward Nucleophiles. J. Am. Chem. Soc. 2011, 133 (13), 4998-5008.

(36) Allongue, P.; Henry de Villeneuve, C.; Morin, S.; Boukherroub, R.; Wayner, D. D. M. The preparation of flat $\mathrm{H}-\mathrm{Si}(111)$ surfaces in 40\% NH4F revisited. Electrochim. Acta 2000, 45 (28), 4591-4598.

(37) Rijksen, B.; Pujari, S. P.; Scheres, L.; van Rijn, C. J. M.; Baio, J. E.; Weidner, T.; Zuilhof, $H$. Hexadecadienyl Monolayers on Hydrogen-Terminated Si(111): Faster Monolayer Formation and Improved Surface Coverage Using the Enyne Moiety. Langmuir 2012, 28 (16), 6577-6588.

(38) Scheres, L.; Giesbers, M.; Zuilhof, H. Organic Monolayers onto Oxide-Free Silicon with Improved Surface Coverage: Alkynes versus Alkenes. Langmuir 2010, 26 (7), 4790-4795.

(39) Scheres, L.; Arafat, A.; Zuilhof, H. Self-Assembly of HighQuality Covalently Bound Organic Monolayers onto Silicon. Langmuir 2007, 23 (16), 8343-8346.

(40) Gangarapu, S.; Pujari, S. P.; Alon, H.; Rijksen, B.; Sukenik, C. N.; Zuilhof, H. Effect of $\alpha$-Heteroatoms on the Formation of AlkeneDerived Monolayers on $\mathrm{H}-\mathrm{Si}(111)$ : A Combined Experimental and Theoretical Study. Langmuir 2015, 31 (30), 8318-8327.

(41) Scheres, L.; Rijksen, B.; Giesbers, M.; Zuilhof, H. Molecular Modeling of Alkyl and Alkenyl Monolayers on Hydrogen-Terminated Si(111). Langmuir 2011, 27 (3), 972-980.

(42) Sieval, A. B.; van den Hout, B.; Zuilhof, H.; Sudhölter, E. J. R Molecular Modeling of Covalently Attached Alkyl Monolayers on the Hydrogen-Terminated Si(111) Surface. Langmuir 2001, 17 (7), 2172-2181.

(43) Pei, Y.; Ma, J.; Jiang, Y. Formation Mechanisms and Packing Structures of Alkoxyl and Alkyl Monolayers on Si(111): Theoretical Studies with Quantum Chemistry and Molecular Simulation Models. Langmuir 2003, 19 (18), 7652-7661.

(44) Glendening, E. D.; et al., NBO Version 3.1.

(45) Frisch, M. J.; et al. Gaussian 09; Gaussian, Inc.: Wallingford, CT, 2009.

(46) Gao, F.; Teplyakov, A. V. Reaction of Hydrazine with a Chlorine-Terminated Si(111) Surface. J. Phys. Chem. C 2014, 118 (48), 27998-28006.

(47) Puniredd, S. R.; Assad, O.; Haick, H. Highly Stable Organic Modification of $\mathrm{Si}(111)$ Surfaces: Towards Reacting Si with Further Functionalities while Preserving the Desirable Chemical Properties of Full Si-C Atop Site Terminations. J. Am. Chem. Soc. 2008, 130 (29), 9184-9185.

(48) Bashouti, M. Y.; Stelzner, T.; Berger, A.; Christiansen, S.; Haick, H. Chemical Passivation of Silicon Nanowires with C1-C6 Alkyl Chains through Covalent Si-C Bonds. J. Phys. Chem. C 2008, 112 (49), 19168-19172.

(49) Nemanick, E. J.; Hurley, P. T.; Brunschwig, B. S.; Lewis, N. S. Chemical and Electrical Passivation of Silicon (111) Surfaces through Functionalization with Sterically Hindered Alkyl Groups. J. Phys. Chem. B 2006, 110 (30), 14800-14808.

(50) Campos, M. A. C.; Paulusse, J. M. J.; Zuilhof, H. Functional monolayers on oxide-free silicon surfaces via thiol-ene click chemistry. Chem. Commun. 2010, 46 (30), 5512-5514.

(51) Wallart, X.; Henry de Villeneuve, C.; Allongue, P. Truly Quantitative XPS Characterization of Organic Monolayers on Silicon: Study of Alkyl and Alkoxy Monolayers on H-Si(111). J. Am. Chem. Soc. 2005, 127 (21), 7871-7878.

(52) Soria, F. A.; Zhang, W.; van Duin, A. C. T.; Patrito, E. M. Thermal Stability of Organic Monolayers Grafted to $\mathrm{Si}(111)$ : Insights from ReaxFF Reactive Molecular Dynamics Simulations. ACS Appl. Mater. Interfaces 2017, 9 (36), 30969-30981.

(53) Carvalho, R. R.; Pujari, S. P.; Lange, S. C.; Sen, R; Vrouwe, E. X.; Zuilhof, H. Local Light-Induced Modification of the Inside of Microfluidic Glass Chips. Langmuir 2016, 32 (10), 2389-2398. 